\title{
Carnot Cycle at Finite Power: Attainability of Maximal Efficiency
}

\author{
Armen E. Allahverdyan, ${ }^{1}$ Karen V. Hovhannisyan, ${ }^{2,1}$ Alexey V. Melkikh, ${ }^{3}$ and Sasun G. Gevorkian ${ }^{4}$ \\ ${ }^{1}$ Yerevan Physics Institute, Alikhanian Brothers Street 2, Yerevan 375036, Armenia \\ ${ }^{2}$ ICFO-Institut de Ciències Fotòniques, Mediterranean Technology Park, 08860 Castelldefels (Barcelona), Spain \\ ${ }^{3}$ Ural Federal University, Mira Street 19, Yekaterinburg 620002, Russia \\ ${ }^{4}$ Institute of Physics, Academia Sinica, Nankang, Taipei 11529, Taiwan
}

(Received 28 March 2013; revised manuscript received 7 June 2013; published 1 August 2013)

\begin{abstract}
We want to understand whether and to what extent the maximal (Carnot) efficiency for heat engines can be reached at a finite power. To this end we generalize the Carnot cycle so that it is not restricted to slow processes. We show that for realistic (i.e., not purposefully designed) engine-bath interactions, the workoptimal engine performing the generalized cycle close to the maximal efficiency has a long cycle time and hence vanishing power. This aspect is shown to relate to the theory of computational complexity. A physical manifestation of the same effect is Levinthal's paradox in the protein folding problem. The resolution of this paradox for realistic proteins allows to construct engines that can extract at a finite power $40 \%$ of the maximally possible work reaching $90 \%$ of the maximal efficiency. For purposefully designed engine-bath interactions, the Carnot efficiency is achievable at a large power.
\end{abstract}

DOI: 10.1103/PhysRevLett.111.050601

PACS numbers: 05.20.-y, 05.10.Gg

Reciprocating heat engines extract work operating cyclically between two thermal baths at temperatures $T_{1}$ and $T_{2}$ $\left(T_{1}>T_{2}\right)$ [1]. They have two basic characteristics: (i) efficiency $\eta=W / Q_{1}$ is the work $W$ extracted per cycle divided by the heat input $Q_{1}$ from the high-temperature bath; (ii) power $W / \tau_{\mathrm{c}}$, where $\tau_{\mathrm{c}}$ is the cycle duration. Both these quantities have to be large for a good engine: if $\eta$ is small, a lot of energy is wasted; if the power is small, no sizable work is delivered over a reasonable time [1].

The second law establishes the Carnot efficiency $\eta_{\mathrm{C}}=$ $1-\left(T_{2} / T_{1}\right)$ as an upper bound for $\eta$ [1]. The Carnot cycle reaches the bounding value $\eta_{C}$ in the (useless) limit, where the power goes to zero [1]. Conversely, realistic engines are not efficient, since they have to be powerful; e.g., the efficiency of Diesel engines amounts to 35\%-40\% of the maximal value. This power-efficiency dilemma motivated a search for the efficiency that would generally characterize the maximal power regime. One candidate for this is the Curzon-Ahlborn efficiency $\eta_{\mathrm{CA}}=1-\sqrt{T_{2} / T_{1}}$ [2], which is, however, crucially tied to the linear regime $T_{1} \approx T_{2}[3,4]$. Beyond this regime, $\eta_{\mathrm{CA}}$ is a lower bound of $\eta$ for a class of model engines [5]. Several recent models for the efficiency at the maximal power overcome $\eta_{\mathrm{CA}}$ with $\eta^{*}=\eta_{\mathrm{C}} / 2-\eta_{\mathrm{C}}[6]$.

As argued in $[5,7,8]$, the maximal power regime allows for the Carnot efficiency, at least for certain models. But it is currently an open question whether the maximal efficiency is attained under realistic conditions (see, e.g., [9] versus [7]), and how to characterize the very realism of those conditions. Even more generally: what is the origin of the power-efficiency dilemma? We answer these questions by analyzing a generalized Carnot cycle, which in contrast to the original Carnot cycle is not restricted to slow processes. We now summarize our answers.
(I) When the $N$-particle engine operates at the maximal work extracted per cycle, its efficiency reaches the Carnot bound $\eta_{\mathrm{C}}$ for $N \gg 1$, while the cycle time is given by the relaxation time of the engine. The maximal work and the Carnot efficiency are achieved due to the flat energy landscape of the engine. For realistic engine-bath interactions this energy landscape leads to a very long $\left[\mathcal{O}\left(e^{N}\right)\right]$ relaxation time nullifying the power. By realistic we mean interactions that are independent from the engine Hamiltonian. If we assume a proper tuning between engine-bath interaction and the engine Hamiltonian, the relaxation time scales as $\mathcal{O}(\sqrt{N})$, and the maximal efficiency is achievable in the limit $N \gg 1$ at a large power $\mathcal{O}(\sqrt{N})$.

(II) The relaxation of the optimal engine under realistic interactions relates to an important problem of searching an unstructured database for a marked item, where each energy level refers to a database item. This task is computationally complex; i.e., even the most powerful quantum algorithms resolve it in $\mathcal{O}\left(e^{N / 2}\right)$ time-steps [10]. Hence the power-efficiency dilemma relates to computational complexity. The same effect can be reformulated as Levinthal's paradox of the protein folding problem: if the majority of unfolded states of a protein are assumed to have the same (free) energy, the folding time is very long [11].

(III) A scenario of resolving Levinthal's paradox proposed in protein science shows the way of constructing suboptimal engines that operate at a reasonably large values of work, power, and efficiency. These suboptimal engines function as model proteins, but they are restricted to mesoscopic scales $N \sim 100$ - otherwise, the relaxation time is again large. Sacrificing some $50 \%-60 \%$ of the maximal possible work leads to reasonable cycle times with the efficiency that achieves some $90 \%$ of the maximal (Carnot) efficiency. 
Carnot cycle and its generalization.-Recall that the Carnot cycle consists of four slow, consecutive pieces [1]: thermally isolated, isothermal, thermally isolated, isothermal. Four times slow brings in the vanishing power stressed above; see additionally section I of [12]. Since the overall process is a quasiequilibrium one, the external fields that extract work from the engine act on it during all the four pieces. One deduces for the isothermal parts: $Q_{1}=T_{1} \Delta S$ and $Q_{2}=T_{2} \Delta S$, where $Q_{1}\left(Q_{2}\right)$ is the heat taken from (put into) the $T_{1}$-bath ( $T_{2}$-bath), and $\Delta S>0$ is the entropy change. Since the work extracted is $W=Q_{1}-$ $Q_{2}, \eta$ equals to its maximal value $\eta_{\mathrm{C}}=1-\left(T_{2} / T_{1}\right)$ [1].

We keep the two isothermal and two thermally isolated pieces of the original Carnot cycle, but do not force them to be slow. In addition, the external fields will act only during the thermally isolated stages. Isothermal pieces amount to free relaxation. Due to these points, we can analyze the engine functioning from the energy conservation. We study the engine via quantum mechanics on a finite Hilbert space, because this reduces the problem to a combinatorial optimization. The final results are interpreted classically and can be also obtained by discretizing the Hamiltonian classical dynamics over phase-space cells.

(i) The engine $E$ with the Hamiltonian $H_{1}$ starts in an equilibrium state at temperature $T_{1}$ described by the density matrix

$$
\rho(0)=\rho_{1}=e^{-\beta_{1} H_{1}} /\left(\operatorname{tr} e^{-\beta_{1} H_{1}}\right), \quad \beta_{1}=1 / T_{1} .
$$

(ii) Between times 0 and $\tau, E$ undergoes a thermally isolated process with a time-dependent Hamiltonian $H_{12}(t)$ and the unitary evolution $\rho(\tau)=U_{12} \rho(0) U_{12}^{\dagger}$ :

$$
\begin{gathered}
H_{12}(0)=H_{1}, \quad H_{12}(\tau)=H_{2}, \\
U_{12}=\mathcal{T} e^{-i \int_{0}^{\tau} d s H_{12}(s)},
\end{gathered}
$$

where $\mathcal{T}$ means chronological ordering. The work taken out of $E$ is determined by energy conservation (see [1] and section II of [12]),

$$
W_{1}=\operatorname{tr}\left[H_{1} \rho_{1}-H_{2} U_{12} \rho_{1} U_{12}^{\dagger}\right] .
$$

(iii) Then, $E$ is attached to the $T_{2}$ bath and after relaxation time $\tau_{\mathrm{r}}$ its density matrix becomes

$$
\rho\left(\tau+\tau_{\mathrm{r}}\right)=\rho_{2}=e^{-\beta_{2} H_{2}} /\left(\operatorname{tr} e^{-\beta_{2} H_{2}}\right) .
$$

The heat that came to $E$ from the $T_{2}$ bath is

$$
Q_{2}=\operatorname{tr}\left[H_{2} \rho_{2}-H_{2} U_{12} \rho_{1} U_{12}^{\dagger}\right] .
$$

(iv) $E$ undergoes another thermally isolated process,

$$
\begin{gathered}
H_{21}(0)=H_{2}, \quad H_{21}(\tau)=H_{1}, \\
U_{21}=\mathcal{T} e^{-i \int_{0}^{\tau} d s H_{21}(s)},
\end{gathered}
$$

completing the cycle with respect to the Hamiltonian. The work taken out of $E$ reads

$$
W_{2}=\operatorname{tr}\left[H_{2} \rho_{2}-H_{1} U_{21} \rho_{2} U_{21}^{\dagger}\right] .
$$

(v) Finally, $E$ is attached to the $T_{1}$-bath $\left(T_{1}>T_{2}\right)$ and relaxes to $\rho_{1}$ thereby completing the cycle; see (1). The heat that came to $E$ from the $T_{1}$-bath is

$$
Q_{1}=\operatorname{tr}\left[H_{1} \rho_{1}-H_{1} U_{21} \rho_{2} U_{21}^{\dagger}\right] .
$$

To stress the differences with the original Carnot cycle: (i) the cycle time $2\left(\tau+\tau_{\mathrm{r}}\right)$ need not be much larger than the relaxation time $\tau_{\mathrm{r}}$; (ii) the cycle is out of equilibrium; and (iii) the work source and the bath never act simultaneously, either one acts or another. Hence, heat and work are deduced from the energy conservation.

We did not count the work necessary for switching the system-bath interaction on and off, because we assume that it does not contribute to the total work budget (e.g., since it is weak).

Maximization of work.-We maximize the full extracted work $W=W_{1}+W_{2}$ over $H_{1}, H_{2}, U_{12}, U_{21}$ for fixed $T_{1}>T_{2}$ and a fixed number $n+1$ of energy levels of $E$. The lowest energies of $H_{1}$ and $H_{2}$ can be set to zero. Introduce the eigenresolution of $H_{\alpha}$ :

$$
H_{\alpha}=\sum_{k=2}^{n+1} \epsilon_{k}^{[\alpha]}\left|k^{[\alpha]}\right\rangle\left\langle k^{[\alpha]}\right|, \quad \alpha=1,2 .
$$

The full work $W=W_{1}+W_{2}$ reads from (3) and (7)

$$
\begin{aligned}
W= & \sum_{\alpha=1}^{2} \sum_{k=2}^{n+1} p_{k}^{[\alpha]} \epsilon_{k}^{[\alpha]} \\
& -\sum_{k, l=2}^{n+1}\left[p_{k}^{[2]} \epsilon_{l}^{[1]} C_{k l}^{[21]}+p_{k}^{[1]} \epsilon_{l}^{[2]} C_{k l}^{[12]}\right],
\end{aligned}
$$

where $\left\{p_{k}^{[\alpha]}\right\}_{k=1}^{n+1}$ are eigenvalues of $\rho_{\alpha}$ given by (1), (4), and (9), and where

$C_{k l}^{[\alpha \gamma]} \equiv\left|\left\langle k^{[\alpha]}\left|U_{\alpha \gamma}\right| l^{[\gamma]}\right\rangle\right|^{2}, \quad(\alpha, \gamma)=(1,2),(2,1)$.

$C_{k l}^{[\alpha \gamma]}$ are doubly stochastic matrices: $\sum_{k=1}^{n+1} C_{k l}^{[\alpha \gamma]}=$ $\sum_{l=1}^{n+1} C_{k l}^{[\alpha \gamma]}=1$. Such a matrix $C_{k l}$ can be represented as a convex sum of permutation matrices (Birkhoff's theorem) [13]: $C_{k l}=\sum_{\delta} \lambda_{\delta} \Pi_{k l}^{[\delta]}$, where $\lambda_{\delta} \geq 0, \sum_{\delta} \lambda_{\delta}=1$, and where $\Pi^{[\delta]}$ permutes the components of any vector on which it acts. Hence, we can maximize $W$ in (10) and (11) over $\left\{\lambda_{\delta}\right\}$. The optimal $C_{k l}^{[12]}$ and $C_{k l}^{[21]}$ amount to permutation matrices, since $\left\{\lambda_{\delta}\right\}$ enter linearly into $W$. Without loss of generality we can assume $\epsilon_{1}^{[\alpha]} \leq \cdots \leq$ $\epsilon_{n+1}^{[\alpha]}$ and hence $p_{1}^{[\alpha]} \geq \cdots \geq p_{n+1}^{[\alpha]}$. Then the optimal permutations $C_{k l}^{[12]}$ and $C_{k l}^{[21]}$ are unit matrices; see (11). In contrast to the original Carnot cycle, the optimal thermally isolated processes can be realized as sudden (quick) changes of the Hamiltonian eigenvalues without changing the eigenvectors. A prominent example of such a process is the Mössbauer effect [14]. It is an advantage that thermally 
isolated processes can be fast; otherwise it is difficult to maintain thermal isolation, which is a known problem of the original Carnot cycle [1].

The work

$$
W=W_{1}+W_{2}=\sum_{k=2}^{n+1}\left(p_{k}^{[1]}-p_{k}^{[2]}\right)\left(\epsilon_{k}^{[1]}-\epsilon_{k}^{[2]}\right),
$$

is to be still maximized over $\left\{\epsilon_{k}^{[1]}\right\}_{k=2}^{n+1}$ and $\left\{\epsilon_{k}^{[2]}\right\}_{k=2}^{n+1}$; see (12). $W$ is symmetric with respect to permutations within $\left\{\epsilon_{k}^{[1]}\right\}_{k=2}^{n+1}$ and within $\left\{\epsilon_{k}^{[2]}\right\}_{k=2}^{n+1}$. We checked numerically that this symmetry is not broken and hence the maximum of $W$ is reached for

$$
\epsilon^{[\alpha]} \equiv \epsilon_{2}^{[\alpha]}=\cdots=\epsilon_{n+1}^{[\alpha]}, \alpha=1,2 ;
$$

i.e., all excited levels have the same energy. Thus, the thermally isolated pieces of the cycle consist, respectively, of sudden changes $\epsilon^{[1]} \rightarrow \epsilon^{[2]}$ and $\epsilon^{[2]} \rightarrow \epsilon^{[1]}$.

With new variables $e^{-\beta_{\alpha} \epsilon^{[\alpha]}} \equiv u_{\alpha}$ we write the maximal work as

$$
W_{\max }\left[u_{1}, u_{2}\right]=\frac{\left(T_{1} \ln \frac{1}{u_{1}}-T_{2} \ln \frac{1}{u_{2}}\right)\left(u_{1}-u_{2}\right) n}{\left[1+n u_{1}\right]\left[1+n u_{2}\right]},
$$

where $u_{1}$ and $u_{2}$ are found from

$$
\partial_{u_{1}} W_{\max }\left[u_{1}, u_{2}\right]=\partial_{u_{2}} W_{\max }\left[u_{1}, u_{2}\right]=0 .
$$

$u_{1}$ and $u_{2}$ depend on $T_{2} / T_{1}$ and on $n$. Noting (8) and the result before (13) we obtain $Q_{1}=\operatorname{tr}\left[H_{1}\left(\rho_{1}-\rho_{2}\right)\right]$ for the heat obtained from the high-temperature bath. Using (14) we get from $\eta=W / Q_{1}$ and from (15):

$$
\eta=1-\left[T_{2} \ln u_{2}\right] /\left[T_{1} \ln u_{1}\right] .
$$

Note from (15) that $W_{\max }\left[u_{1}, u_{2}\right]>0$ and $T_{2}<T_{1}$ imply $1>\epsilon^{[2]} / \epsilon^{[1]}>T_{2} / T_{1}$. Hence, (17) implies $\eta \leq \eta_{\mathrm{C}}=$ $1-T_{2} / T_{1}$, as expected.

Both $W_{\max }\left[u_{1}, u_{2}\right]$ and $\eta$ increase with $n$. For $\ln [n] \gg 1$ we get asymptotically from (16):

$$
u_{1}=\frac{(1-\theta) \ln [n]}{n}, \quad u_{2}=\frac{\theta}{n \ln [n](1-\theta)},
$$

where $\theta \equiv T_{2} / T_{1}$. This produces

$$
\begin{gathered}
W_{\max }\left[u_{1}, u_{2}\right]=\left(T_{2}-T_{1}\right) \ln [n]-\mathcal{O}(1 / \ln [n]), \\
\eta=\eta_{\mathrm{C}}-\mathcal{O}(1 / \ln [n]), \quad \eta_{\mathrm{C}} \equiv 1-T_{2} / T_{1} .
\end{gathered}
$$

The maximal work $W_{\max }\left[u_{1}, u_{2}\right]$ scales as $\ln [n]$, since this is the "effective number of particles" for the engine. In the macroscopic limit $\ln [n] \gg 1$, the efficiency converges to its maximal value $\eta_{\mathrm{C}}=1-T_{2} / T_{1}$; see (20).

The cycle time.- -amounts to two times the relaxation time $\tau_{\mathrm{r}}$ of the system with spectrum (14) and energy gap $\epsilon \sim \ln [n]$; see (14) and (18). (Recall that the thermally isolated stages of the cycle are very quick.) The magnitude of $\tau_{\mathrm{r}}$ essentially depends on the scenario of relaxation.
First (specific) scenario.-We can assume that the Hamiltonian (9) and (14) of the heat engine is known. Then there exist system-bath interaction scenarios that generally produce a non-Markovian dynamics of the system and lead to $\tau_{\mathrm{r}}=\mathcal{O}(\sqrt{\ln [n]})$; see sections VI and VII of [12]. Hence for this type of relaxation the Carnot efficiency is achievable at a large power $\mathcal{O}(\sqrt{\ln [n]}) \gg 1$; see (19). However, in these scenarios the system-bath interaction Hamiltonian (that governs the relaxation) is special: it depends on the engine Hamiltonian (9) and (14). [If also the bath Hamiltonian is tailored, the relaxation time can be reduced to $\mathcal{O}(1)$ reaching the power $\mathcal{O}(\ln [n])$.]

Second (realistic) scenario.-Assuming that the systembath interaction does not depend on the Hamiltonian (9) and (14), we can estimate $\tau_{\mathrm{r}}$ within the weak-coupling, Markov master equation approach that leads to $\tau_{\mathrm{r}}=\mathcal{O}(n)$; see section III of [12]. For a qualitative understanding of this situation, consider the relaxation as a random walk in the energy space, e.g. in the second step of the cycle, where the engine starts with almost unpopulated ground state, and it has to achieve ground state probability $\approx 1$ after relaxation; see (18). So, if every transition from one excited energy level to another takes a finite time, one will need to perform in average $n / 2$ transitions before jumping to the ground state. Now note from (20) that the convergence of $\eta$ to $\eta_{\mathrm{C}}$ is controlled by $\mathcal{O}(1 / \ln [n])$ : a small step towards $\eta_{\mathrm{C}}$ will lead to a large increase in $\tau_{\mathrm{r}}$ nullifying the power $\mathcal{O}(\ln [n] / n)$ for $n \gg 1$; see (19). Hence for this type of relaxation the Carnot efficiency is not achievable at a finite power.

The second relaxation scenario of the system with Hamiltonian (9) and (14) is similar to the known combinatorial optimization problem: finding a marked item in an unstructured database [10] of $n+1$ items. This problem is mapped to physics by associating each item to an eigenvector of a Hamiltonian [10]. The marked item relates to the lowest energy level 0 , while all other (excited) eigenvalues of the Hamiltonian $\epsilon$ are equal. The resulting system has unknown eigenvectors of the Hamiltonian, but known eigenenergies. Now the searching process can be organized as a relaxation of the system from an excited state to a low-temperature equilibrium state. This state is dominated by the ground level due to a large $\epsilon$. Once the relaxation is over, the unknown item (eigenvector) can be revealed by measuring the energy [15].

For classical algorithms the search time of this problem scales as $\mathcal{O}(n)$ for $n \gg 1$ [10]. It is thus not much better than going over all possible candidates for the solution, a typical situation of a computationally complex problem. For quantum algorithms (Grover's search) the search time scales as $\mathcal{O}(\sqrt{n})$ [10]. This is still not suitable for our purposes, since it nullifies the power for $\ln [n] \gg 1$.

Suboptimal engine.-Within the second (realistic) relaxation scenario, we shall modify the optimal engine so that the power is finite, but both the work and efficiency are still large. We are guided by the analogy between the 
relaxation of the Hamiltonian (9) and (14) under the second scenario and Levinthal's paradox from protein physics [11]. In fact, (9) and (14) is the simplest model employed for illustrating the paradox; see [11,17] and section V of [12]. Here the ground state refers to the unique folded (native) state. To ensure its stability, it is separated by a large gap from excited (free) energy levels. The essence of the paradox is that assuming many equivalent unfolded (excited) states, the relaxation time to the native state is unrealistically long. Recall that the states $\rho_{1}$ and $\rho_{2}$ of the optimal engine refer, respectively, to unfolded and folded states of the protein model. Indeed, $n u_{\alpha} /\left(1+n u_{\alpha}\right)$ $(\alpha=1,2)$ is the overall probability of the excited levels; see (14). Hence, for $\ln [n] \gg 1$ the ground state (excited levels) dominates in $\rho_{2}\left(\rho_{1}\right)$; see (18).

The resolution of the paradox is to be sought via resolving the degeneracy of excited levels: if there are energy differences, some (unfavorable) transitions will not be made shortening the relaxation time $[11,17]$. In resolving the energy degeneracy we follow the simplest model proposed in [11].

The model has $N \gg 1$ degrees of freedom $\left\{\sigma_{i}\right\}_{i=1}^{n}$; each one can be in $\zeta+1$ states: $\sigma_{i}=0, \ldots, \zeta$. Whenever $\sigma_{i}=0$ for all $i$ 's, the model protein is in the folded (ground) state with energy zero $[11,17]$. The ground state has zero energy. Excited states with $s \geq 1$ have energy $\epsilon+\delta s$, where $\epsilon>0$ and $s$ is the number of (misfolded) degrees of freedom with $\sigma_{i} \neq 0 . \delta>0$ is the parameter that (partially) resolves the degeneracy of excited states; we revert to the previous, work-optimal, model for $\delta \rightarrow 0$. For different eigenvalues of the Hamiltonian $H_{\alpha}$ we have

$$
\left\{\left(1-\delta_{\mathrm{Kr}}[s, 0]\right)\left(\epsilon^{[\alpha]}+s \delta^{[\alpha]}\right)\right\}_{s=0}^{N}, \quad \alpha=1,2,
$$

where $\delta_{\mathrm{Kr}}[s, 0]$ is the Kronecker delta, and where each energy $\epsilon^{[\alpha]}+s \delta^{[\alpha]}$ is degenerate $\zeta^{s} N ! / s !(N-s)$ ! times; thus, the total number of energy levels is $(1+\zeta)^{N}$.

Given (21), the cycle consists of two isothermal and two thermally isolated pieces with sudden changes $\left(\delta^{[1]}, \epsilon^{[1]}\right) \rightarrow$ $\left(\delta^{[2]}, \epsilon^{[2]}\right) \rightarrow\left(\delta^{[1]}, \epsilon^{[1]}\right)$; see (1)-(13). Below, we shall also assume

$$
\beta_{1} \delta^{[1]}=\beta_{2} \delta^{[2]},
$$

because this makes the suboptimal engine structurally very similar to the optimal one. Now the work $W=W_{1}+W_{2}$ is calculated from (3), (7), (8), (21), and (22):

$$
\begin{gathered}
W\left[v_{1}, v_{2} ; K\right]=\frac{m\left(\Delta \epsilon+\frac{K N \Delta \delta}{1+K}\right)\left(v_{1}-v_{2}\right)}{\left(1+m v_{1}\right)\left(1+m v_{2}\right)}, \\
\Delta \epsilon=\epsilon^{[1]}-\epsilon^{[2]}=T_{1} \ln \left[1 / v_{1}\right]-T_{2} \ln \left[1 / v_{2}\right], \\
\Delta \delta=\delta^{[1]}-\delta^{[2]}=\left(T_{1}-T_{2}\right) \ln [\zeta / K],
\end{gathered}
$$

where $K=\zeta e^{-\beta_{1} \delta^{[1]}}, m=(1+K)^{N}$, and where $v_{\alpha} \equiv$ $e^{-\beta_{\alpha} \epsilon^{[\alpha]}}(\alpha=1,2)$ are determined from maximizing (23).
TABLE I. Parameters of the sub-optimal engine: work $W$, efficiency $\eta$, and the cycle time $2 \tau_{\mathrm{r}}$; see (23) and (27). $W_{\max }$ is the maximal work extracted for the optimal engine at a vanishing power; see (15) and (16). For the suboptimal engine: $K=\zeta e^{-\beta_{1} \delta^{[1]}}, N=140, \zeta=4, T_{1}=1, T_{2}=1 / 2$. Carnot and Curzon-Ahlborn efficiencies are, respectively, $\eta_{\mathrm{C}}=1 / 2$ and $0.5858 \eta_{\mathrm{C}}$. Also, $p_{1}^{[\alpha]}=\left[1+(1+K)^{N} e^{-\beta_{\alpha} \epsilon^{[\alpha]}}\right]^{-1}(\alpha=1,2)$ are the ground-state probabilities of $\rho_{\alpha} \propto e^{-\beta_{\alpha} H_{\alpha}} ;$ see (21).

\begin{tabular}{lcccccc}
\hline \hline$K$ & $\tau_{\mathrm{r}}$ & $W / W_{\max }$ & $W$ & $\eta / \eta_{\mathrm{C}}$ & $p_{1}^{[1]}$ & $p_{1}^{[2]}$ \\
\hline 0.1 & $4.45 \times 10^{-5} \mathrm{~s}$ & 0.2267 & 23.52 & 0.8751 & 0.0392 & 0.9808 \\
0.2 & $4.35 \mathrm{~s}$ & 0.3884 & 40.3 & 0.9110 & 0.0237 & 0.9883 \\
0.24 & $357 \mathrm{~s}$ & 0.4393 & 45.58 & 0.9181 & 0.0210 & 0.9896 \\
\hline \hline
\end{tabular}

Note the analogy between (15) and (23), with $m$ being an analogue of $n$; they get equal for $\delta \rightarrow 0$. Note that in (23) we neglected factor $\mathcal{O}(1 / m)$ assuming that $m \gg 1$.

Likewise, we get for the efficiency [cf. (17)]:

$$
\eta=1-\frac{T_{2}}{T_{1}} \times \frac{\ln \frac{1}{v_{2}}+\frac{N K \ln (\zeta / K)}{1+K}}{\ln \frac{1}{v_{1}}+\frac{N K \ln (\zeta / K)}{1+K}} .
$$

For this model [11] assumes a local Markov relaxation dynamics, where each degree of freedom makes a transition $\sigma_{i} \rightarrow \sigma_{i} \pm 1$ in $10^{-9}$ seconds; this value is chosen conventionally to fit experimental magnitudes for the elementary dynamic step [11]. The model has a single relaxation time [11] that is easily reproduced in the general master equation framework (see section IV of [12]):

$$
\tau_{\mathrm{r}}=10^{-9}(1+K)^{N} /(N K) \text { seconds, }
$$

where the factor $N$ is due to the $N$-times degenerate first excited level.

For $\delta^{[\alpha]} \rightarrow 0(\alpha=1,2)$, where the excited energy levels become degenerate, $\tau_{\mathrm{r}} \propto(1+\zeta)^{N}$ scales linearly over the number of energy levels, as expected. When $\delta^{[\alpha]}$ are not zero, $\tau_{\mathrm{r}}$ can be of order of 1 second for $N \sim 100$, because $1+K$ is close to 1. However, for the macroscopic situation $\left(N \sim 10^{23}\right) \tau_{\mathrm{r}}$ is still huge. In this sense, the model is incomplete, but still useful for analyzing the mesoscopic situation $N \sim 100$ that is relevant for the protein folding problem [17].

Table I illustrates the characteristics of the sub-optimal engine and compares them with those of the optimal one. Reasonable cycle times can coexist with a finite fraction $(\sim 40 \%)$ of the maximal work and with sizable efficiencies ( $\sim 90 \%$ of the maximal value) that are larger than the Curzon-Ahlborn value. Hence, albeit within the second (realistic) scenario it is impossible to approach the maximal efficiency as close as desired, reasonably large efficiencies at a finite (or even large) power are possible. These results resemble the power-efficiency trade-off (see [18]), but they are more complicated, since they involve work, efficiency, and power.

K. V.H. is supported by the Spanish project FIS201014830. S. G. G. is supported by Grant No. NSC 101-2811M-001-156. 
[1] H. B. Callen, Thermodynamics (John Wiley, New York, 1985).

[2] J. Yvon, Proceedings of the International Conference on Peaceful uses of Atomic Energy, United Nations, Geneva, 1955, p. 387; P. Chambadal, Les Centrales Nucléaires (Armand Colin, Paris, France, 1957); I. I. Novikov, J. Nucl. Energy 7, 125 (1958); F. Curzon and B. Ahlborn, Am. J. Phys. 43, 22 (1975).

[3] C. Van den Broeck, Phys. Rev. Lett. 95, 190602 (2005).

[4] M. Esposito, K. Lindenberg, and C. Van den Broeck, Phys. Rev. Lett. 102, 130602 (2009).

[5] A. E. Allahverdyan, R. S. Johal, and G. Mahler, Phys. Rev. E 77, 041118 (2008)

[6] See, e.g., M. Esposito, R. Kawai, K. Lindenberg, and C. Van den Broeck, Phys. Rev. Lett. 105, 150603 (2010).

[7] G. Benenti, K. Saito, and G. Casati, Phys. Rev. Lett. 106, 230602 (2011).

[8] U. Seifert, Phys. Rev. Lett. 106, 020601 (2011).

[9] K. Brandner, K. Saito, and U. Seifert, Phys. Rev. Lett. 110, 070603 (2013).

[10] E. Farhi and S. Gutmann, Phys. Rev. A 57, 2403 (1998).
[11] R. Zwanzig, Proc. Natl. Acad. Sci. U.S.A. 92, 9801 (1995).

[12] See Supplemental Material at http://link.aps.org/ supplemental/10.1103/PhysRevLett.111.050601 for details.

[13] A. W. Marshall and I. Olkin, Inequalities: Theory of Majorization and its Applications (Academic Press, New York, 1979).

[14] A. B. Migdal, Qualitative Methods in Quantum Theory (Benjamin, London, 1977).

[15] Since the full Hamiltonian is not known, this measurement will at least take $\hbar /(\delta \epsilon)$ seconds due to the energy-time uncertainty principle, where $\delta \epsilon$ is the precision by which the ground-state energy is measured; see [16]. This time is, however, a small overhead to the search time.

[16] Y. Aharonov, S. Massar, and S. Popescu, Phys. Rev. A 66, 052107 (2002).

[17] K. Sneppen and G. Zocchi, Physics in Molecular Biology (Cambridge University Press, Cambridge, England, 2005).

[18] B. Jiménez de Cisneros and A. C. Hernández, Phys. Rev. Lett. 98, 130602 (2007). 